\title{
Velocidade de corrida associada ao consumo máximo de oxigênio em meninos de 10 a 15 anos
}

\author{
Fabiana Andrade Machado ${ }^{1}$, Luiz Guilherme Antonacci Guglielmo ${ }^{2}$ e Benedito Sérgio Denadai ${ }^{1}$
}

\section{RESUMO}

O objetivo deste estudo foi determinar a influência da idade cronológica e da maturação biológica sobre o consumo máximo de oxigênio $\left(\dot{\mathrm{V}}_{2 \max }\right)$ e a velocidade de corrida correspondente ao $\mathrm{VO}_{2 \max }$ em crianças e adolescentes brasileiros, do sexo masculino, com idade entre 10 e 15 anos, não praticantes de atividade física sistemática. Participaram do estudo 40 voluntários, divididos em dois grupos, segundo a idade cronológica $(\mathrm{GC} 1-\mathrm{n}=20 ; 11,4 \pm 0,6$ anos; $38,8 \pm 8,6 \mathrm{~kg} ; 143,6 \pm 8,2 \mathrm{~cm}$ e GC2 $-\mathrm{n}=20 ; 14,1 \pm$ $0,6$ anos; $55,9 \pm 14,2 \mathrm{~kg} ; 163,3 \pm 10,2 \mathrm{~cm})$ e maturação biológica (GB1 $-\mathrm{n}=20$; estágios 1,2 e 3 ; e GB2 $-\mathrm{n}=20$; estágios 4 e 5). $\mathrm{O} \mathrm{VO}_{2 \max }$ foi mensurado em um teste progressivo e intermitente de corrida em esteira rolante, com estágios de três minutos e pausa de 20 segundos, incrementos de $1 \mathrm{~km} / \mathrm{h}$ a começar com $9 \mathrm{~km} / \mathrm{h}$, até a exaustão voluntária. A velocidade correspondente ao $\dot{\mathrm{VO}}_{2 \max }\left(\mathrm{v} \dot{\mathrm{VO}}_{2 \max }\right)$ foi considerada como a menor velocidade em que se observou o maior valor de $\mathrm{V}_{2}$. A máxima velocidade aeróbia $\left(\mathrm{Va}_{\max }\right)$ foi calculada pela fórmula proposta por di Prampero (1986). Houve diferença significante para os valores de $\dot{\mathrm{V}}{ }_{2 \max }(1 / \mathrm{min}), \mathrm{Va}_{\text {max }}(\mathrm{km} / \mathrm{h})$ e $\mathrm{vV}_{2 \max }(\mathrm{km} / \mathrm{h})$ entre os grupos GC1 e GC2 $(1,84 \pm 0,41 / 2,81 \pm 0,61 ; 11,8 \pm 1,2$ / 12,6 $\pm 1,2 ; 12,1 \pm 1,2 / 12,9 \pm 1,1$, respectivamente), GB1 e GB2

1. Laboratório de Avaliação da Performance Humana, Unesp, Rio Claro SP.

2. Laboratório de Avaliação do Esforço Físico, Fefil, Lins - SP.

Recebido em: 3/8/2001

Aceito em: 20/11/2001

Endereço para correspondência:

Benedito Sérgio Denadai

Laboratório de Avaliação da Performance Humana

Instituto de Biociências

Av. 24A, 1.515 - Bela Vista

13506-900 - Rio Claro, SP - Brasil

E-mail: bdenadai@rc.unesp.br
$(1,80 \pm 0,37 / 2,87 \pm 0,56 ; 12,1 \pm 1,2 / 12,9 \pm 1,1 ; 11,8 \pm$ 1,2 / 12,5 $\pm 1,1$, respectivamente), mas não para os valores

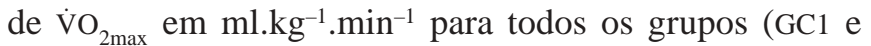
GC2: 47,9 \pm 6,8 / 50,4 $\pm 5,5$; GB1 e GB2: 47,9 \pm 6,8 / 50,3 \pm 5,5 , respectivamente). Com base nos resultados obtidos, pode-se concluir que o $\dot{\mathrm{V}}_{2 \max }(\mathrm{l} / \mathrm{min})$, a $\mathrm{Va}_{\max }$ e a $\mathrm{vV}_{2 \max }$ têm seus valores aumentados como um provável efeito do crescimento e desenvolvimento, podendo, ainda, expressar melhora da economia de movimento, mesmo em indivíduos não praticantes de atividade física sistemática.

Palavras-chave: Consumo máximo de oxigênio. Máxima velocidade aeróbia. Crianças. Adolescentes.

\section{ABSTRACT}

\section{Running speed associated with maximal oxygen uptake in boys aged 10 to 15 years}

The purpose of this study was to determine the influence of chronological age and biological maturation on the maximal oxygen uptake $\left(\dot{V} O_{2 \max }\right)$ and the speed corresponding to the $\dot{V} O_{2 \max }$ in Brazilian boys aged 10-15 years under no regular training program. Forty volunteers participated in this study. They were divided into groups by chronological age $(\mathrm{GCl}-n=20 ; 11.45 \pm 0.63$ years; $38.8 \pm 8.6 \mathrm{~kg}$; $143.6 \pm 8.2 \mathrm{~cm}$; and $G C 2-n=20 ; 14.1 \pm 0.6$ years; $55.9 \pm$ $14.2 \mathrm{~kg} ; 163.3 \pm 10.2 \mathrm{~cm})$ and by biological maturation (GB1 $-n=20$; stages 1, 2, 3, and GB2 $-n=20$; stages 4, 5). The $\dot{V} O_{2 \max }$ was determined with a progressive and intermittent test in a treadmill, starting from a speed of $9 \mathrm{~km} /$ $h$ and increasing $1 \mathrm{~km} / \mathrm{h}$ each 3 minutes until volunteer exhaustion. The speed at $\dot{V} O_{2 \max }\left(v \dot{V} O_{2 \max }\right)$ was the lowest speed that elicited $\dot{V} O_{2 \max }$ (peak value). The Va $a_{\max }$ was calculated in accordance with di Prampero (1986). There was a significant difference of $\dot{V} O_{2 \max }(\mathrm{l} / \mathrm{min}), \mathrm{Va}_{\max }(\mathrm{km} / \mathrm{h})$ and $v \dot{V} O_{2 \max }(\mathrm{km} / \mathrm{h})$ between $\mathrm{GCl}$ and $\mathrm{GC2}(1.84 \pm 0.41 / 2.81 \pm$ $0.61 ; 11.8 \pm 1.2 / 12.6 \pm 1.2 ; 12.1 \pm 1.2 / 12.9 \pm 1.1$, respectively); $G B 1$ and $G B 2(1.8 \pm 0.37 / 2.87 \pm 0.56 ; 12.1$ $\pm 1.2 / 12.9 \pm 1.1 ; 12.5 \pm 1.1$, respectively), but there was 
no significant difference of $\dot{V} O_{2 \max }\left(\mathrm{ml} \mathrm{kg}^{-1} \cdot \mathrm{min}^{-1}\right)$ between groups (GCl and GC2; GB1 and GB2). The authors can conclude that the $\dot{V} O_{2 \max }, V a_{\max }$ and $v \dot{V} O_{2 \max }$ had their values increased probably by growth and development processes, and can also express a better economy of movement, even in individuals not involved in a regular training program.

Key words: Maximal oxygen uptake. Maximal aerobic speed. Children. Adolescents.

\section{INTRODUÇÃO}

Durante a atividade física, o organismo altera o seu funcionamento na tentativa de manter essa atividade pelo maior período de tempo. Para que essa manutenção seja possível, é essencial que haja maior oferta de oxigênio aos tecidos, em especial, àqueles que estão sendo exaustivamente exigidos. Diminuição na oferta de oxigênio pode comprometer a realização de uma atividade física.

O consumo máximo de oxigênio $\left(\dot{\mathrm{V}}_{2 \max }\right)$ é o índice que melhor representa, quantitativa e qualitativamente, a capacidade funcional do sistema cardiorrespiratório durante a atividade física, sendo considerado o método padrão-ouro entre todos os índices ${ }^{1,2}$. É definido como a mais alta captação de oxigênio alcançada por um indivíduo, respirando ar atmosférico ao nível do mar $^{3}$. Caracteriza-se pela perfeita integração do organismo em captar, transportar e utilizar oxigênio para os processos aeróbios de produção de energia durante esforço físico ${ }^{4}$. Em repouso, o $\mathrm{VO}_{2}$ mostrase similar em indivíduos sedentários e treinados. No entanto, durante o esforço físico máximo, os valores encontrados em indivíduos treinados são visivelmente maiores em relação aos sedentários ${ }^{1,4}$.

Outras diferenças são observadas também quando comparamos as respostas de $\dot{\mathrm{V}}_{2 \max }$ obtidas em crianças, adolescentes e adultos. Quando expresso em valores absolutos (1/min), o $\dot{\mathrm{VO}}_{2 \max }$ aumenta dos 8 aos 18 anos. Tal aumento pode chegar a $11 \%$ ao ano. Isso se deve a fatores relacionados ao próprio crescimento e desenvolvimento, como o aumento nas dimensões das estruturas corporais e, também, ao grande envolvimento em atividades físicas nesse período da vida ${ }^{5}$. Observam-se, ainda, diferenças nos valores de $\dot{\mathrm{V}}_{2 \max }$ obtidos em meninos e meninas de mesma faixa etária, tanto expresso em valores absolutos ( $1 / \mathrm{min})$ quanto em valores relativos à massa corporal $\left(\mathrm{ml} \cdot \mathrm{kg}^{-1} \cdot \mathrm{min}^{-1}\right)$. Por volta dos 13 anos, os meninos demonstram aumento da massa muscular, enquanto as meninas apresentam aumento do tecido adiposo. Como o $\dot{\mathrm{VO}}_{2 \max }$ é dependente da massa muscular envolvida na atividade ${ }^{6}$, existe aumento proporcional à idade nos meninos e estabilização ou mesmo declínio de seus valores nas meninas. Bar-Or ${ }^{7}$ obser- vou que, aos oito anos de idade, essa diferença entre meninos e meninas é de aproximadamente $16 \%$, aos 14 anos é de $25 \%$ e aos 16 anos, de $50 \%$.

Outra variável utilizada para predizer a performance, principalmente em eventos de longa duração, é a economia de movimento, sendo denominada, na corrida, de economia de corrida (EC). Pode ser definida como o custo de oxigênio ( $\left.\mathrm{V}_{2}\right)$ para uma dada atividade de intensidade submáxima ${ }^{4}$. Sua utilização mostra-se eficaz ao determinar a performance, especialmente em provas de longa duração, ainda que, para os indivíduos envolvidos na atividade, haja valores similares de $\mathrm{V}_{2 \max }{ }^{8}$. A EC também é influenciada pela idade cronológica. MacDougall et al. ${ }^{9}$ realizaram um estudo transversal com 134 indivíduos de 7 a 16 anos e verificaram que o $\dot{\mathrm{VO}}_{2}\left(\mathrm{ml} \mathrm{kg}^{-1} \cdot \mathrm{min}^{-1}\right)$, obtido em fase estável durante diferentes velocidades de corrida $\left(7,5 \mathrm{a} 16 \mathrm{~km} \cdot \mathrm{h}^{-1}\right)$, decrescia em relação à idade cronológica. Em um estudo longitudinal realizado em crianças sem atividade física sistemática, verificou-se aumento da EC com o avançar da idade; e que as crianças mais econômicas aos 10 anos mantiveram-se assim por volta dos 17 anos, o mesmo ocorrendo em relação às menos econômicas ${ }^{10}$. Como o $\dot{V}_{2 \max }$ $\left(\mathrm{ml} \cdot \mathrm{kg}^{-1} \cdot \mathrm{min}^{-1}\right)$ em meninos entre 10 e 18 anos não se altera significativamente, parte da melhora na performance aeróbia de corrida observada nesta fase pode ser justificada pela melhora da EC proporcionada pelo processo de crescimento.

Segundo alguns autores, a identificação da intensidade de esforço correspondente ao $\mathrm{V}_{2 \max }$ é a melhor maneira de descrever a associação entre a potência aeróbia máxima e a $\mathrm{EC}^{11,12}$. A $v \dot{V}_{2 \max }$ é definida como a mínima velocidade em que há ocorrência do $\mathrm{VO}_{2 \max }$, sendo considerada um importante preditor de performance aeróbia e controlador dos efeitos do treinamento ${ }^{13}$. Billat et al. ${ }^{14}$ verificaram que o $\dot{\mathrm{VO}}_{2 \max }\left(\mathrm{ml} \mathrm{kg}^{-1} \cdot \mathrm{min}^{-1}\right)$ e a $v \dot{\mathrm{VO}}{ }_{2 \max }(\mathrm{km} / \mathrm{h})$ encontrados para meninos de 10 a 12 anos foram significantemente maiores do que para as meninas de mesma faixa etária $(49,4$ / $40,4 \mathrm{ml} / \mathrm{kg} / \mathrm{min} ; 12,6 / 11,2 \mathrm{~km} / \mathrm{h}$, respectivamente). A maior $\mathrm{VVO}_{2 \max }$ dos meninos pode ser atribuída em parte aos maiores valores de $\dot{\mathrm{VO}}_{2 \max }\left(\mathrm{ml} \mathrm{kg}^{-1} \cdot \mathrm{min}^{-1}\right) \mathrm{e}$, também, às diferenças de EC entre os gêneros ${ }^{14}$.

Poucos ainda são os estudos que analisaram os efeitos da idade cronológica e da maturação biológica sobre a $v \dot{V} O_{2 \max }$. Do mesmo modo, não se encontram estudos que tenham medido diretamente o $\dot{\mathrm{VO}}_{2 \max }$ em crianças brasileiras sem prática de atividade física sistemática, havendo apenas autores que o fizeram através de métodos indire$\operatorname{tos}^{15}$.

Com isso, o objetivo deste estudo foi determinar os efeitos da idade cronológica e da maturação biológica sobre o $\dot{\mathrm{V}} \mathrm{O}_{2 \max }$ e a sua respectiva velocidade, em crianças e adoles- 
centes brasileiros do sexo masculino, com idade entre $10 \mathrm{e}$ 15 anos, não praticantes de atividade física sistemática.

\section{MATERIAL E MÉTODOS}

\section{Sujeitos}

Fizeram parte deste estudo 40 crianças e adolescentes do sexo masculino, com idade entre 10 e 15 anos, aparentemente saudáveis, não participantes de qualquer programa sistemático de treinamento físico. Antes de iniciarem os testes, seus responsáveis tomaram conhecimento dos procedimentos aos quais as crianças seriam submetidas e assinaram o termo de concordância referente à participação no estudo. Todos os procedimentos foram aprovados pelo Comitê de Ética em Pesquisa do IB/Unesp - Rio Claro. Posteriormente, os participantes foram distribuídos em dois grupos, segundo a idade cronológica, sendo considerada a data de nascimento e a data de realização do teste para calcular a idade centesimal de cada um $(\mathrm{GC1}-10,5$ a 12,4 anos e GC2 - 12,5 a 15,4 anos).

\section{Maturação biológica}

A maturação biológica foi determinada através da maturação sexual, segundo os índices padronizados por Tanner ${ }^{16}$. Observou-se o desenvolvimento das características sexuais secundárias (pilosidade pubiana), classificando os indivíduos em categorias de 1 a 5 , de acordo com padrões fotográficos. O exame foi realizado em todos os participantes pelo mesmo examinador. Posteriormente, os participantes foram distribuídos em dois grupos, segundo o nível de maturação: GB1 - estágios 1, 2 e 3; GB2 - estágios 4 e 5 .

\section{Antropometria}

As dobras cutâneas das regiões tricipital (TR) e subescapular (SE) foram determinadas (Cescorfe) sempre do lado direito do voluntário. Foram realizadas três mensurações em cada região, assumindo-se como valor final a média desses valores. Todo o procedimento foi realizado por um mesmo avaliador para que fosse minimizada a ocorrência de erros. A predição da percentagem de gordura foi realizada segundo a fórmula proposta por $\mathrm{Lohman}^{17}: \% \mathrm{G}=1,35$ $(\Sigma$ TR $+\mathrm{SE})-0,012(\Sigma \text { TR }+\mathrm{SE})^{2}-\mathrm{C}$, em que C é uma constante dependente da idade cronológica.

\section{Consumo máximo de oxigênio}

$\mathrm{O} \dot{\mathrm{VO}}_{2 \max }$ foi determinado em um teste laboratorial de corrida em esteira rolante (Inbramed Super ATL), utilizando-se para isso um protocolo progressivo e intermitente. A velocidade de aquecimento foi de $5 \mathrm{~km} / \mathrm{h}$ e o primeiro estágio foi a $9 \mathrm{~km} / \mathrm{h}$, sendo mantida inclinação de $1 \%$ durante todo o teste. $\mathrm{O}$ incremento de velocidade foi de $1 \mathrm{~km} / \mathrm{h}$ a cada estágio até a exaustão voluntária, porém, por estímulo verbal, o participante foi encorajado a permanecer na atividade pelo maior tempo possível. Cada estágio teve a duração de três minutos, com 20 segundos de pausa entre os mesmos para a coleta de amostras de sangue do lóbulo da orelha $(25 \mu \mathrm{l})$ para posterior análise da concentração de lactato sanguíneo (YSI 1500 STAT).

A medida da captação de gases foi realizada por um sistema de ergoespirometria de circuito aberto (Aerosport Teem 100), cujo funcionamento é válido e fidedigno ${ }^{18,19}$, fornecendo informações sobre o comportamento respiratório a cada 20 segundos. Previamente ao início do teste, cada participante permaneceu em repouso por 15 minutos, estando em pé e já acoplado ao equipamento de captação de gases para a determinação do $\mathrm{V}_{2}$ de repouso.

Adotou-se como $\dot{\mathrm{V}}_{2 \max }$ o valor mais alto de consumo obtido durante o teste, verificando, também, outras reações que pudessem confirmar sua ocorrência: vermelhidão facial, hiperventilação, freqüência cardíaca $(\mathrm{FC}) \geq 200 \mathrm{bpm}$, descompasso de passadas e razão de trocas respiratórias (R) $\geq 1,0$.

A FC foi constantemente monitorada através de um freqüencímetro (Polar Vantage XL).

Determinação da velocidade correspondente ao $\mathrm{V}_{2 \max }$ ( $\left(v \dot{V} O_{2 \text { max }}\right)$ e a máxima velocidade aeróbia $\left(\mathrm{Va}_{\max }\right)$

A intensidade correspondente ao $\mathrm{V}_{2 \max }$ tem sido determinada por diferentes critérios e terminologias ${ }^{4,20}$. Neste estudo, foram determinadas a $\mathrm{vV}_{2 \max }$ e a $\mathrm{Va}_{\max }$ (máxima velocidade aeróbia). A vVंO ${ }_{2 \max }$ foi considerada como sendo a menor velocidade em que foi observado o maior valor $\mathrm{VO}_{2}{ }^{13}$. A Va ${ }_{\max }$ foi calculada pela fórmula proposta por di Prampero ${ }^{11}: \mathrm{Va}_{\max }=\left(\dot{\mathrm{V}}_{2 \max }-\dot{\mathrm{V}}_{2}\right.$ rep $) \times \mathrm{CR}-1$, em que CR é o custo de energia por unidade de massa corporal, referente a uma velocidade específica (submáxima), calculado pela fórmula: $\mathrm{CR}=\left(\mathrm{V}_{2} \mathrm{v}-\dot{\mathrm{V}} \mathrm{O}_{2} \mathrm{rep}\right) \mathrm{x} \mathrm{v}^{-1} \mathrm{em} \mathrm{mlO} / \mathrm{kg}^{-1} \mathrm{~min}^{-1}$, sendo que esta velocidade (v) é referente a $75 \%$ do $\dot{\mathrm{V}}_{2 \max }$.

\section{Análise estatística}

O dados estão expressos em média \pm desvio padrão. $\mathrm{O}$ teste $t$ para dados não pareados foi utilizado para comparar os valores encontrados para os dois grupos (GB1 x GB2 e GC1 x GC2). Em todos os testes adotou-se um nível de significância de $\mathrm{p} \leq 0,05$.

\section{RESULTADOS}

Na tabela 1 encontram-se os valores médios referentes às características antropométricas dos participantes distribuídos de acordo com os grupos (GC1, GC2, GB1 e GB2). A 
idade, massa corporal e estatura foram significantemente maiores nos grupos $\mathrm{GC} 2$ e GB2 em relação aos grupos $\mathrm{GC} 1$ e GB1, respectivamente. Não foi observada diferença para o percentual de gordura entre os grupos ( $\mathrm{GC1}$ x GC2 e GB1 x GB2).

Na tabela 2 encontram-se os valores médios para $\dot{\mathrm{V}}_{2 \max }(1 / \mathrm{min}$ e ml.kg-1 $\left.\mathrm{min}^{-1}\right), \mathrm{Va}_{\max }, \mathrm{vV}_{2 \max }$ e lactato pico dos participantes (GC1, GC2, GB1 e GB2). Houve diferença estatisticamente significante para o $\dot{\mathrm{VO}}_{2 \max }(\mathrm{l} / \mathrm{min})$, $\mathrm{Va}_{\max }$ e $\mathrm{vV \dot { }}{ }_{2 \max }$ de GC2 em relação a GC1, e de GB2 em relação a GB1. O $\dot{\mathrm{V}}_{2 \max }$ em valores relativos $\left(\mathrm{ml}^{\mathrm{kg}} \mathrm{kg}^{-1} \cdot \mathrm{min}^{-1}\right)$ e o pico de lactato após o teste incremental não foram diferentes entre os grupos.

Quando realizamos um ajuste exponencial para normalização do $\dot{V}^{2}{ }_{2 \max }$ em relação à massa corporal $\left(\mathrm{ml} \cdot \mathrm{kg}^{0,67} \cdot \mathrm{min}^{-1}\right)$, que, segundo alguns autores ${ }^{21,22}$, remove as diferenças de dimensão corporal que podem interferir nos resultados, observamos diferença estatisticamente significante entre os valores encontrados entre $\mathrm{GC} 1 \mathrm{e}$ GC2 $\left(158,4 \pm 22,3\right.$ e $190,2 \pm 19,7 \mathrm{ml} \cdot \mathrm{kg}^{0,67} \cdot \mathrm{min}^{-1}$, respectivamente).

Da amostra total $(n=40)$, observamos em três crianças $(7,5 \%)$ aumento menor que $2 \mathrm{mlO}_{2} \mathrm{~kg}^{-1} \cdot \mathrm{min}^{-1}$ entre o último minuto do penúltimo e último estágios. Segundo alguns autores ${ }^{23,24}$, esse comportamento pode caracterizar a ocorrência de platô de $\dot{\mathrm{V}}_{2}$. Ainda que esse comportamento tenha sido observado em pequena parte da amostra, 31 indivíduos apresentaram valores de $\mathrm{R} \geq 1,0(77,5 \%)$ e nove, valores $0,9<\mathrm{R}<1,0$. Todas as crianças da amostra apresentaram frequiência cardíaca máxima (FCM) com valores equivalentes ou maiores que $95 \%$ do valor máximo predito para a idade (220 - idade). Em relação ao pico de lactato sanguíneo após o teste incremental, apenas dois indivíduos $(5 \%)$ apresentaram valores maiores que $8 \mathrm{mM}$.

A figura 1 exemplifica a relação entre o $\dot{\mathrm{V}}_{2}$ e a velocidade de corrida para um indivíduo de $\mathrm{GC} 1$ e outro de $\mathrm{GC} 2$, que apresentam o mesmo valor de $\dot{\mathrm{VO}}_{2 \max }\left(\mathrm{ml} \cdot \mathrm{kg}^{-1} \cdot \mathrm{min}^{-1}\right) \mathrm{e}$ diferentes $\mathrm{Va}_{\max }$, revelando maior EC para o indivíduo mais velho.

* p $<0,05$ em relação a GC1

* $p<0,05$ em relação a $\mathrm{G} 1$

** $p<0,05$ em relação a GB1
TABELA 1 grupos divididos de acordo com a idade cronológica GC1 e GC2) e maturação biológica (GB1 e GB2)

\begin{tabular}{lrrrr}
\hline \multicolumn{1}{c}{ Variáveis } & GC1 (n=20) & GC2 (n=20) & GB1 (n=20) & GB2 (n= 20) \\
& & & & \\
Idade (anos) & $11,4 \pm 0,6$ & $14,1 \pm 0,6^{*}$ & $11,6 \pm 0,9$ & $13,9 \pm 0,8^{* *}$ \\
Massa corporal (kg) & $38,8 \pm 8,6$ & $55,9 \pm 14,2^{*}$ & $37,9 \pm 7,7$ & $56,9 \pm 13,4^{* *}$ \\
Altura (cm) & $143,6 \pm 8,2$ & $163,3 \pm 10,2^{*}$ & $142,8 \pm 7,2$ & $164,2 \pm 9,1^{* *}$ \\
\% Gordura & $15,5 \pm 9,8$ & $14,9 \pm 5,7$ & $16,0 \pm 9,4$ & $14,4 \pm 6,2$ \\
\hline
\end{tabular}

Valores médios \pm DP para o consumo máximo de oxigênio $\left(\mathrm{V}_{2 \max }\right)$,

TABELA 2 suas respectivas velocidades $\left(\mathrm{Va}_{\max }\right.$ e $\left.\mathrm{VVO}_{2 \max }\right)$ e o lactato pico (mM) dos grupos divididos de acordo com a idade cronológica (GC1 e GC2) e maturação biológica (GB1 e GB2)

\begin{tabular}{|c|c|c|c|c|}
\hline Variáveis & GC1 $(n=20)$ & GC2 (n = 20) & GB1 $(n=20)$ & GB2 $(n=20)$ \\
\hline$\dot{\mathrm{VO}}_{2 \max }(\mathrm{l} / \mathrm{min})$ & $1,84 \pm 0,4$ & $2,81 \pm 0,6 *$ & $1,80 \pm 0,3$ & $2,87 \pm 0,5^{*}$ \\
\hline$\dot{\mathrm{V}} \mathrm{O}_{2 \max }(\mathrm{ml} / \mathrm{kg} / \mathrm{min})$ & $47,9 \pm 6,8$ & $50,4 \pm 5,5$ & $47,9 \pm 6,8$ & $50,3 \pm 5,5$ \\
\hline $\mathrm{Va}_{\max }(\mathrm{km} / \mathrm{h})$ & $11,8 \pm 1,2$ & $12,6 \pm 1,23^{*}$ & $12,1 \pm 1,2$ & $12,9 \pm 1,1 * *$ \\
\hline $\mathrm{VVO}_{2 \max }(\mathrm{km} / \mathrm{h})$ & $12,1 \pm 1,2$ & $12,9 \pm 1,13^{*}$ & $11,8 \pm 1,2$ & $12,5 \pm 1,1 * *$ \\
\hline Lactato pico (mM) & $4,1 \pm 1,7$ & $4,3 \pm 2,0$ & $4,1 \pm 1,6$ & $4,3 \pm 2,1$ \\
\hline
\end{tabular}

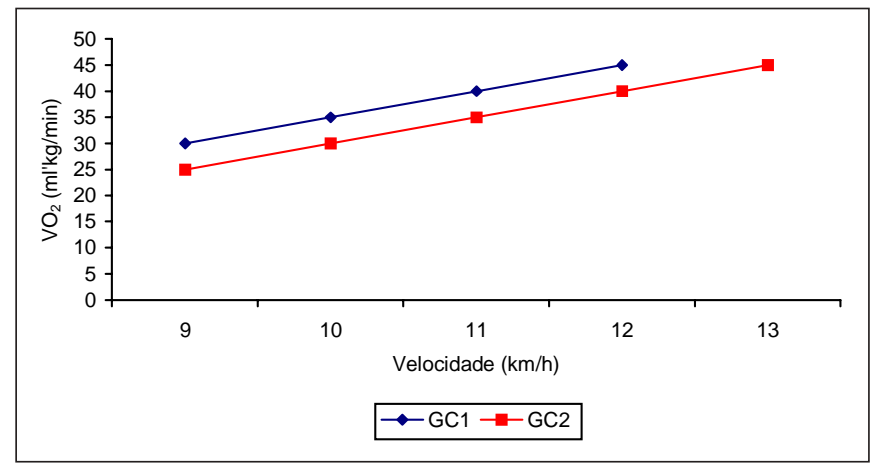

Fig. 1 - Relação entre o consumo de oxigênio $\left(\dot{V} \mathrm{O}_{2}\right)$ e a velocidade de corrida para um indivíduo do GC1 (10,5 a 12,4 anos) e outro do GC2 (12,5 a 15,4 anos), que apresentam o mesmo valor de $\dot{V} O_{2 \max }(\mathrm{ml} / \mathrm{kg} /$ min) e diferentes velocidades máximas aeróbias $\left(V a_{\max }\right)$.

\section{DISCUSSÃO}

O objetivo deste estudo foi analisar os efeitos da idade cronológica e da maturação biológica sobre o $\mathrm{VO}_{2 \max }$ e suas respectivas velocidades $\left(\mathrm{Va}_{\max }\right.$ e $\left.\mathrm{vV}_{2 \max }\right)$ em crianças e adolescentes brasileiros do sexo masculino, não participantes de qualquer programa de treinamento sistemático. Con- 
cordando com estudos anteriores ${ }^{25,26}$, verificou-se que o $\dot{\mathrm{V}}_{2 \max }$ em valores absolutos ( $\left.\mathrm{l} / \mathrm{min}\right)$ aumenta, enquanto que, em valores relativos $\left(\mathrm{ml} \cdot \mathrm{kg}^{-1} \cdot \mathrm{min}^{-1}\right)$, se mantém constante dos 10 aos 15 anos de idade em meninos não treinados. Embora exista essa manutenção dos valores relativos, as intensidades associadas ao $\mathrm{V}_{2 \max }$ determinadas por dois diferentes critérios aumentaram nessa faixa etária, sugerindo melhora da EC com o aumento da idade.

Testes realizados com o objetivo de mensurar o $\dot{\mathrm{V}}_{2 \max }$ em crianças podem apresentar certa restrição quanto à validade e fidedignidade dos resultados obtidos ${ }^{27}$. O comportamento que classicamente assegura a obtenção do $\dot{\mathrm{V}}_{2 \max }$ é a ocorrência do platô (aumento da intensidade sem modificação dos valores de $\mathrm{V}_{2}$ ) durante o teste incremental. Estudos $^{23,27}$ revelam que pequena percentagem $(30 \%)$ de crianças apresenta a ocorrência desse fenômeno. Em relação aos adultos, sua ocorrência perfaz em média 50\% dos testes realizados ${ }^{6}$. Alguns autores sugerem que aumentos no $\dot{\mathrm{V}}_{2}$ menores que $2 \mathrm{ml} \cdot \mathrm{kg}^{-1} \cdot \mathrm{min}^{-1}$ ou $150 \mathrm{ml} / \mathrm{min}$, entre o último minuto do penúltimo e do último estágio do teste incremental, podem caracterizar a ocorrência do platôô 23,24 . Entretanto, estudos recentes ${ }^{23,27}$, que analisaram um número bastante elevado de crianças e adolescentes, demonstraram que, embora somente a minoria dos indivíduos apresentasse um platô de $\dot{\mathrm{V}}_{2}$, não foram encontradas diferenças hemodinâmicas (freqüência cardíaca máxima), metabólicas ( $\mathrm{R}$ e lactato sanguíneo) e antropométricas (estatura e massa corporal) entre os indivíduos que atingiram ou não o platô de $\mathrm{V}_{2}$. Além disso, verificou-se que os indivíduos que não mostraram o platô de $\dot{\mathrm{V}}_{2}$ possuíam no $\dot{\mathrm{V}}_{2}$ pico seus valores máximos, pois, quando submetidos a esforços supramáximos, não exibiram aumento do $\dot{\mathrm{V}}_{2}$, a despeito de apresentarem elevação dos índices ( $\mathrm{R}$ e lactato sanguíneo), que indicam crescimento da contribuição anaeróbia nessas condições ${ }^{23}$. A ausência do platô em crianças pode ocorrer pela sua menor capacidade anaeróbia e, também, pela sua menor capacidade de motivação e concentração em relação aos adultos ${ }^{5,7}$.

Em função disso, alguns parâmetros têm sido propostos para que seja aceito como $\dot{\mathrm{V}}_{2 \max }$ o valor de pico encontrado no teste. Entre os parâmetros mais aceitos, temos a FCM $\geq 200 \mathrm{bpm} ; \mathrm{R} \geq 1,0$; hiperventilação, vermelhidão facial; descompasso de passadas 24,28 . Apesar de termos observado em apenas três crianças $(7,5 \%)$ aumento de $\mathrm{V}_{2}$ menor que $2 \mathrm{ml} \cdot \mathrm{kg}^{-1} \cdot \mathrm{min}^{-1}$ entre o último minuto do penúltimo e último estágios (o que pode caracterizar a ocorrência de platô), 77,5\% da amostra total $(\mathrm{n}=31)$ apresentou $\mathrm{R} \geq 1,0 \mathrm{e}$ todos os participantes demonstraram FCM $\geq$ a $95 \%$ do valor máximo predito para a idade, o que nos levou a aceitar o maior valor observado durante o teste (pico) como sendo o $\dot{\mathrm{VO}}_{2 \max }$.

Rev Bras Med Esporte - Vol. 8, № 1 - J an/ Fev, 2002
Outro aspecto que dificulta a validade dos testes de $\mathrm{V}_{2 \text { max }}$ realizados em crianças é a escassez de equipamentos e protocolos que sejam especialmente desenvolvidos para verificar as respostas ao exercício nessa população especial ${ }^{27}$. Especificamente em relação aos protocolos, Rivera-Brown et al. ${ }^{28}$ não encontraram diferença significante entre os valores obtidos com a utilização de protocolo contínuo e intermitente, em testes destinados a mensurar o $\dot{\mathrm{V}}_{2 \max } \mathrm{em}$ crianças.

Em estudos longitudinais realizados em crianças e adolescentes europeus, japoneses, norte-americanos e canadenses, verificou-se aumento contínuo nos valores de $\dot{\mathrm{V}}_{2 \max }$ (1/min) entre os 8 e 18 anos de idade, com aumento médio de $11 \%$ ao ano ${ }^{25-27}$. Esse comportamento também foi observado em nosso estudo, sendo os valores muito próximos aos encontrados pelos autores citados anteriormente. Fatores como o aumento nas dimensões das estruturas corporais e, também, o grande envolvimento em atividades físicas nesse período da vida são apontados como responsáveis por esses incrementos ${ }^{5}$.

No entanto, quando o $\mathrm{V}_{2 \max }$ é expresso em valores relativos à massa corporal $\left(\mathrm{ml} \cdot \mathrm{kg}^{-1} \cdot \mathrm{min}^{-1}\right)$, as diferenças entre as faixas etárias se mostram discretas e valores estáveis para crianças e adolescentes do sexo masculino entre $8 \mathrm{e}$ 18 anos de idade, como observados em nosso estudo, são freqüentemente encontrados ${ }^{5}$. Esse comportamento sugere, em princípio, que os incrementos do $\dot{\mathrm{V}}_{2 \max }$ ocorreriam basicamente em função do aumento das dimensões corporais (melhora quantitativa), sem modificações qualitativas em função da idade, dos sistemas funcionais envolvidos na determinação do $\dot{\mathrm{VO}}_{2 \max }$. Entretanto, autores como Nevill et al. ${ }^{21} \mathrm{e}$ Welsman et al. ${ }^{22}$ sustentam que a normalização do $\dot{V}_{2 \max }$ pela massa corporal não é apropriada quando se comparam indivíduos com diferentes dimensões corporais, particularmente durante o processo de crescimento e desenvolvimento. Welsman et al. ${ }^{22}$, empregando um ajuste log-linear $\left(\mathrm{ml} \cdot \mathrm{kg}^{0,67} \cdot \mathrm{min}^{-1}\right)$, encontraram valores muito próximos ao verificados em nosso estudo $(\mathrm{G} 1=158,4$ $\left.\pm 22,3 \mathrm{ml} \cdot \mathrm{kg}^{0,67} \cdot \mathrm{min}^{-1} \mathrm{e} \mathrm{G} 2=190,2 \pm 19,7 \mathrm{ml} \cdot \mathrm{kg}^{0,67} \cdot \mathrm{min}^{-1}\right)$ em grupos de mesma faixa etária. Com base nesses resultados, os autores têm proposto que a maturação determina melhora qualitativa das funções fisiológicas e, conseqüentemente, melhora da capacidade funcional dos indivíduos. Entre essas mudanças, temos o aumento da capacidade de oferta de oxigênio (aumento da concentração de hemoglobina) e da área de seção transversa do músculo esquelético.

Em relação aos valores encontrados para $\mathrm{Va}_{\max }(\mathrm{km} / \mathrm{h})$ em GC1 e GC2 (11,8 $\pm 1,2$ e 12,6 $\pm 1,2)$ e GB1 e GB2 $(12,1 \pm$ 1,2 e $12,9 \pm 1,1$ ), verificamos que esses são semelhantes aos encontrados por Billat et al. ${ }^{14} \mathrm{em}$ meninos de 12,2 anos 
em estágio maturacional entre 2 e $3(12,6 \pm 0,2 \mathrm{~km} / \mathrm{h})$, que empregaram o mesmo critério para determinar a velocidade associada ao $\dot{\mathrm{VO}}_{2 \max }\left(\mathrm{Va}_{\max }\right)$. A intensidade correspondente ao $\dot{\mathrm{V}}_{2 \max }$ tem sido determinada por diferentes critérios e terminologias ${ }^{12,20}$. Hill e Rowell ${ }^{20}$ apontam que os critérios empregados por Billat et al. ${ }^{13}\left(\mathrm{vV}_{2 \max }\right)$ envolvem um componente anaeróbio para a determinação da intensidade associada ao $\dot{\mathrm{V}}_{2 \max }$, pois esta intensidade está acima do limiar anaeróbio. Já os critérios propostos por di Prampero $^{11}\left(\mathrm{Va}_{\max }\right)$ determinam intensidades que podem ser teoricamente sustentadas apenas pelo metabolismo aeróbio (embora $100 \%$ do $\dot{V}_{2 \max }$ não possa ser atingido sem participação anaeróbia). Como em nosso estudo os valores de $\dot{V}^{2 \max }\left(\mathrm{ml} \mathrm{kg}^{-1} \cdot \mathrm{min}^{-1}\right)$ não foram diferentes entre os grupos, mas as velocidades associadas ao $\mathrm{V}_{2 \max }$, particularmente a $\mathrm{Va}_{\max }$, foram diferentes, observamos provável melhora na EC com a maturação. A melhora da EC nessa faixa etária tem sido atribuída à diminuição do equivalente ventilatório do oxigênio $\left(\mathrm{VE} / \mathrm{VO}_{2}\right)$, reduzindo o gasto energético da musculatura respiratória ${ }^{5}$, e ao aumento dos membros inferiores, permitindo maior amplitude e diminuição da frequiência das passadas ${ }^{29}$.

Com base nesses resultados, podemos concluir que a idade cronológica e a maturação biológica determinam aumento do $\mathrm{VO}_{2 \max }$ em valores absolutos ( $1 / \mathrm{min}$ ), sem, no entanto, modificar seus valores relativos à massa corporal $\left(\mathrm{ml} . \mathrm{kg}^{-1} \cdot \mathrm{min}^{-1}\right)$. Entretanto, quando se levam em consideração as diferenças nas dimensões corporais $\left(\mathrm{ml} \cdot \mathrm{kg}^{-0,67} \cdot \mathrm{min}^{-1}\right)$, a maturação parece determinar melhora qualitativa da capacidade funcional aeróbia dos indivíduos. As intensidades associadas ao $\dot{\mathrm{VO}}_{2 \max }\left(\mathrm{Va}_{\text {max }}\right.$ e $\left.\mathrm{VVO}_{2 \text { max }}\right)$ aumentam sem modificação do $\dot{V}_{2 \max }\left(\mathrm{ml} \cdot \mathrm{kg}^{-1} \cdot \mathrm{min}^{-1}\right)$, sugerindo melhora da EC em função da idade e da maturação, mesmo em indivíduos que não participam de um programa sistemático de treinamento físico.

\section{REFERÊNCIAS}

1. Astrand PO, Rodhal K. Tratado de fisiologia do exercício. Rio de Janeiro: Guanabara, 1987

2. Shephard RJ, Allen C, Benade AJ, Davies CT, di Prampero PE, Hedman $\mathrm{R}$. The maximum oxygen intake. An international reference standard of cardiorespiratory fitness. Bull World Health Organ 1968;38:757-64.

3. Astrand PO. Human physical fitness with special reference to sex and age. Physiol Rev 1956;36:307-36.

4. Denadai BS. Índices fisiológicos de avaliação aeróbia. Conceitos e aplicações. Ribeirão Preto: BSD, 1999.

5. Rowland TW. Oxygen uptake and endurance fitness in children: a developmental perspective. Pediatr Exerc Sci 1989;1:313-28.

6. Howley ET, Bassett DR, Welch HG. Criteria for maximal oxygen uptake: review and commentary. Med Sci Sports Exerc 1995;27:1292-301.

7. Bar-Or. Pediatric sports medicine for the practitioner. New York: Springer-Verlag, 1983:311-4.
8. Morgan DW, Martin PE, Krahenbuhl GS. Factors affecting running economy. Sports Med 1989;7:310-30.

9. MacDougall JD, Roche PD, Bar-Or O, Moroz JR. Maximal aerobic capacity of Canadian schoolchildren: prediction based on age-related oxygen cost of running. Int J Sports Med 1983;4:194-8.

10. Krahenbuhl GS, Morgan DW, Pangrazi RP. Longitudinal changes in distance-running performance of young males. Int J Sports Med 1989;10: 92-6.

11. Di Prampero PE. The energy cost of human locomotion on land and in water. Int J Sports Med 1986;7:55-72.

12. Denadai BS. Avaliação aeróbia: consumo máximo de oxigênio ou resposta do lactato sanguíneo. In: Avaliação aeróbia: determinação indireta da resposta do lactato sanguíneo. Denadai BS (org.). Rio Claro: Motrix, 2000:1-24.

13. Billat V, Pinoteau J, Petit B, Renoux JC, Koralsztein P. Time to exhaustion at $100 \%$ of velocity at $\dot{\mathrm{VO}}_{2 \max }$ and modeling of the relation timelimit/velocity in elite long distance runners. Eur J Appl Physiol 1994; 69:271-3.

14. Billat V, Gratasdelamarche A, Monnier M, Delamarche P. A test to approach maximal lactate steady-state in 12-year old boys and girls. Arch Physiol Biochem 1995;103:65-72.

15. Duarte CR, Silva MF. Capacidade aeróbica em escolares de 10 a 18 anos: $\dot{V O}_{2 \max }$ e PWC 170. Rev Bras Cienc Mov 1989;3:17-25.

16. Tanner JM. Growth and adolescence. Oxford: Blackwell, 1962.

17. Lohman TG. Applicability of body composition techniques and constants for children and youths. Exerc Sport Sci Rev 1986;14:325-57.

18. Novitsky S, Segal KR, Chatr-Aryamontri B, Guvakov D, Katch VL. Validity of a new portable indirect calorimeter: the AeroSport Teem 100. Eur J Appl Physiol 1995;70:462-7.

19. Wideman L, Stoudemire NM, Pass KA, McGinnes CL, Gaesser GA, Weltman A. Assessment of the aerosport Teem 100 portable metabolic measurement system. Med Sci Sports Exerc 1996;28:509-15.

20. Hill DW, Rowell AL. Running velocity at $\dot{\mathrm{VO}}_{2 \max }$. Med Sci Sports Exerc 1996;28:114-9.

21. Nevill AM, Holder RL. Scaling, normalizing, and per ratio standards: an allometric modeling approach. J Appl Physiol 1995;79:1027-31.

22. Welsman JR, Armstrong N, Nevill AM, Winter EM, Kirby BJ. Scaling peak $\dot{V O}_{2}$ for differences in body size. Med Sci Sports Exerc 1996;28: 259-65.

23. Armstrong $\mathrm{N}$, Welsman $\mathrm{J}$, Winsley R. Is peak $\dot{\mathrm{VO}}_{2}$ a maximal index of children's aerobic fitness? Int J Sports Med 1996;17:356-9.

24. Mahon AD, Marsh ML. Ventilatory threshold and $\dot{\mathrm{VO}}_{2}$ plateau at maximal in 8- to 11-year old children. Pediatr Exerc Sci 1993;5:332-8.

25. Shephard RJ. Influence of added activity classes upon the working capacity of Quebec school children. In: Lavallée H, Shephard RJ, editors. Limites de la capacité physique chez l'enfant. Quebec: Editions du Pelicar, 1977:233-7.

26. Armstrong N, Williams J, Balding J, Gentle P, Kirby B. The peak oxygen uptake of British children with reference to age, sex and sexual maturity. Eur J Appl Physiol 1991;62:369-75.

27. Armstrong N, Welsman JR. Assessment and interpretation of aerobic fitness in children and adolescents. Exerc Sport Sci Rev 1994;22:43576.

28. Rivera-Brown AM, Rivera AM, Frontera WR. Applicability of criteria for $\dot{V}_{2 \max }$ in active adolescents. Pediatr Exerc Sci 1992;4:331-9.

29. Unnithan VB, Eston RG. Stride frequency and submaximal treadmill running economy in adults and children. Pediatr Exerc Sci 1990;2:14955 